\title{
Edge image quality assessment: a new formulation for degraded edge imaging
}

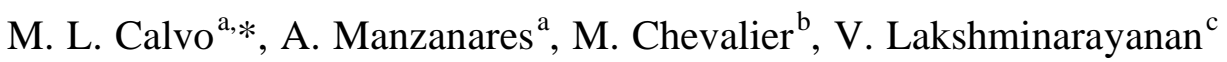 \\ ${ }^{a}$ Departamento de Óptica, Facultad de Ciencias Físicas, Universidad Complutense, 28040 Madrid, Spain \\ ${ }^{\mathrm{b}}$ Física Médica, Departamento de Radiología, Facultad de Medicina, Universidad Complutense, 28040 Madrid, Spain \\ ${ }^{\mathrm{c}}$ University of Missouri-St Louis, School of Optometry and Department of Physics and Astronomy, 8001 Natural Bridge Road, St Louis, MO 63121-4499, USA
}

Received 6 May 1997; received in revised form 18 December 1997; accepted 9 January 1998

\begin{abstract}
We discuss a formalism to characterize degraded edge images formed in a diffraction limited system (with circular pupil of unit radius) under conditions of incoherent illumination. We introduce a novel definition of degraded edges and consider this approach to model a basic optical mechanism involved in the perception of visual depth and edge detection. We introduce a degradation parameter to quantize the degree of edge blur. We present a generalization of such a procedure by assuming the Heaviside function to be a systematic generator of degraded edges. We reproduce experimentally the predictions made by the formalism proposed herein. (C) 1998 Elsevier Science B.V. All rights reserved.
\end{abstract}

Keywords: Image processing; Signal processing; Vision; Edge detection

\section{Introduction}

During the past 40 years, the mathematical framework for edge representation has been extensively analyzed in different branches of optics and related areas such as digital image, signal processing, pattern recognition, robotic vision and artificial intelligence [1,2]. The models were mainly applied to the theory of edge detection, to characterize a specific system by the edge spread function $(E S F)$ or edge trace [3], and for feature extraction and image enhancement by applying an edge detection procedure. Specifically, numerous algorithms have been proposed in digital image processing for image quality improvement in a wide variety of applications (image enhancement, edge and line location, boundary definition, etc.). The majority of the existing algorithms for detection and localization of details and their edges rely substantially on the ideas of optimal linear filters [4].

In the theory of edge detection edges are defined as discontinuities in the image intensity due to changes in the scene structure. These discontinuities originate from different scene features. In digital image processing important contributions have been made in the past decades to solve this problem, especially by implementing edge localization techniques. For example, Bergholm [5] has designed a procedure by applying a gradual focusing algorithm based on a gaussian blurring operator.
A complex mechanism is required to explain the edge signal processing by the visual system. The high demand for edge imaging quality assessment has produced recently several interesting contributions such as the ones from Kayargadde and Martens [6-8] in which they relate the human visual system for edge perception mechanism and a computational model by defining a psychometric space to characterize the computational images and a perceptual space that characterizes the edge detection human performance. A blur index estimation algorithm was developed by introducing an intrinsic blur parameter of the visual system. On the other hand, the experiments by Shapley and Tolhurst [9] and Kulikowski and King-Smith [10] (among others) suggest that there are edge detector mechanisms in the human visual system with a rather uniform efficiency over a wide range of spatial frequencies. Marr and Hildreth [11] proposed a theory of early information processing in complex visual systems, based on the detection of zero-crossing points associated with any edge (binary output response) and its second derivatives. They referred the zero-crossing as the values in the convolution of the image intensity with a mask formed by the application of a laplacian operator on a two-dimensional gaussian distribution. The zero values would correspond to the location of abrupt intensity changes on the image and could be considered as indicators of edges.

In the context of optical image processing, the incoherent (white illumination: absence of interference phenomena), and coherent (light amplitude correlation: interferences

\footnotetext{
* Corresponding author.
} 
background), hard edge imaging is a classical subject with very well known results [12], that accounts for diffraction and propagation phenomena as a consequence of the Rayleigh's limit application (light diffraction by the aperture pupil of the system). One defines the $E S F$, line spread function $(L S F)$ and modulation transfer function $(M T F)$ as well as the $L S F / M T F$ reciprocal relationship introduced by Marchand [13] for linear and invariant perfect systems having rotational symmetry of revolution. Characterizing a system by the ESF definition [14-16] is an alternative procedure to the use of the point spread function $(P S F)$. To enhance the differences one argues that in an ESF characterization Young's theory applies while in the PSF, Huygen's theory is the basis.

Our intention in the present work is to introduce a general procedure to represent systematically various types of degraded edge-objects dealing with the real conditions for an optical instrument to perform an output response, which can be detected by the human visual system or by an experimental set up. We describe a method to characterize the edge degradation in an edge image processing system (EIPS): a diffracted limited system having a circular pupil of unit radius, without aberrations and under normal conditions of illumination (incoherent: absence of interference background noise). Assuming that a degrading process of the edge-object takes place in the system, the present formalism proposes a judgment (edge image quality assessment) of the image quality in terms of its contrast reduction or edge quality degradation. Our main goal in this analysis is to establish a mathematical formalism to represent real edges through which one could account for the influence of significant parameters. We consider in the present analysis not only hard perfect edges at the input of the system, but also non-symmetrical degraded ones, which have a specific degree of blur or defocusing and a non-symmetrical shape. We consider 'degraded edges' since in real systems, the object is not necessarily symmetric or perfectly sharp: real images have edges (or boundaries) with an amount of degradation or blur that depends, for example, on its position of depth in the scene, or may be on a degradation mechanism intrinsic to the edgeobject (for example, a degradation due to atmospheric agents action or any other external agent degrading the edge quality).

The novelty proposed in this paper comes from the mathematical representation for edge degradation. Earlier contributions on this subject [14,15] suppose a degrading optical system that images a hard edge, that is, a system characterized by a degrading impulse response ( $L S F$ or $P S F$ ). Otherwise, we consider a perfect system (characterized by a perfect $L S F$ or $P S F$ ), solely limited by diffraction (being the first effect on degrading the image as considered from Rayleigh's limit) and assume that the input edge function is affected by a degree of blur. Then, the performance of the diffracted limited system at the output is analyzed. It is obvious that real-life vision systems introduce blur (as observed in earlier experiments [11]), but in order to study the edge blur, we start up by considering the $L S F$ of a perfect system.
In Section 2 we present a background on the analytical formulation for perfect edge imaging and degraded edge representation under incoherent illumination. Section 3 contains specific formulation for the intensity transmission function associated with the object and its corresponding image intensity distribution. We introduce the edge degradation as a modified Heaviside function or step function affected by an exponential factor or gradient that accounts for the degradation [17]. We present new ideas in Section 4: the $L S F$ and MTF associated with a degraded edge. These two functions are derived by using the previous definition of degraded edge. By differentiating the image intensity distribution of the degraded edge one obtains an associated $L S F$. The $M T F$ is directly derived by taking the modulus of the Fourier transform of the associated $L S F$. Since these two functions have valid connection to blur, they are both affected by a degradation parameter. In particular, the $L S F$ provides a natural procedure for establishing resolution criteria for degraded edges, while the associated $M T F$ defines a spatial frequency carrier operating under a degradation mechanism (a spatial frequency domain analysis) giving a contrast factor in terms of blur. In Section 5, we introduce a contrast reduction function in terms of the MTF associated with a degraded edge and the MTF of the perfect system. We develop this idea by explaining the basic concept and further applications, as the calculus of the system depth of focus. Section 6 gives some experimental data obtained for real edges captured by a CCD camera and showing the possible connections with the proposed models. We end with Section 7 for discussion and conclusions.

\section{Background: perfect and degraded edge definitions}

In the classical paper by Weinstein [14] it is proposed the use of the incoherent hard edge image distribution to introduce an image quality criterion for incoherent illumination in high aberrated systems (up to $\lambda / 4$ ). This overcomes the PSF definition that shows a complex behavior (fringes structure) and it can be only applied to low aberrated systems (under $\lambda / 4$ ). This author did not consider blur influence or degradation on the edge and introduced a similar parameter to the associated $L S F$, namely, the flux density gradient, which it is a measure of the image quality in the case of simple defocusing.

Barakat and Houston [15] expressed the ESF in terms of an accumulative $L S F$ for a system in the presence of off-axis aberrations. In their treatment, they define the ESF as the hard edge image. Swanter and Hayslett [16] formulated the edge responses for such obscured aperture optical systems as the integral of the line response, considering a perfect edge as object.

In their pioneering work Shanmugam et al. [1] defined the edge as a great and sudden change in an image attribute, usually the brightness. They consider not only the hard edges but the degraded or blurred ones, defined as sigmoids. 


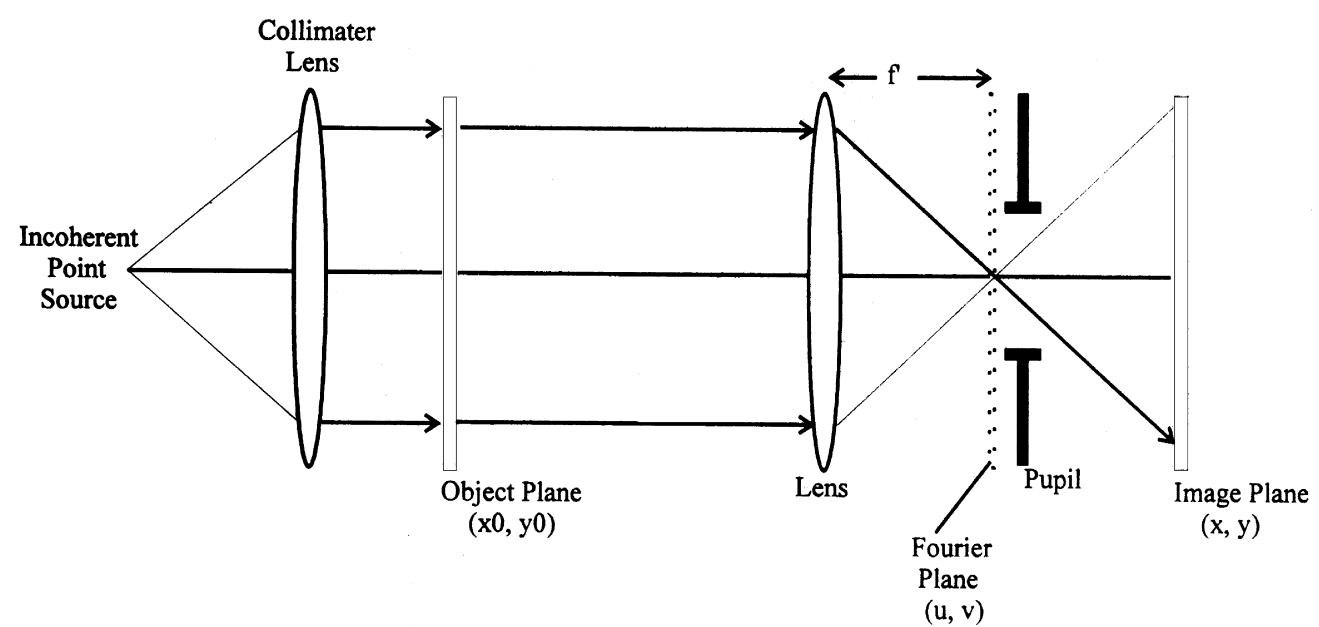

Fig. 1. An image forming system. It is limited by the diffraction of the telecentric effective circular pupil, having unit radius. Incoherent conditions of illumination are assumed.

The sigmoid function has good properties of symmetry, but it does not represent real edges, since they are non-symmetrical due to effects arising from the capturing procedure.

In their theory of edge detection, Marr and Hildreth [11] proposed a theory related to the early processing of visual information in complex vision systems. They considered any sharp intensity change in the image as the presence of an edge. They detected the changes by searching for the zero-crossing of the second derivative of the image intensity distribution. No specific expression for the image intensity distribution was supposed, but the task of isolated edges detection is made by comparing the obtained magnitudes with that for the hard edges.

In an interesting paper, Pentland [18] proposed a new cue for depth information based on the use of perfect edge discontinuities. Pentland calculated the image of the edge by convolving the hard edge object with the $P S F$ of the system, but in the case of defocused edges, Pentland's procedure does not include a blurred magnitude on the edge itself, but in the PSF of the system.

Related to Pentland's contributions, Marshall et al. [19] proposed other cues to relative visual depth based on the perception of edges. The edges were defined as steps, but convolved with a gaussian blur kernel, representing the degrading $L S F$ of the system. The edge image can be defocused or not, depending on this kernel and not on the edge itself.

We find a different definition of a real edge in a paper of Basu [2], where it is defined as a combination of steps, peaks and roofs. It also considers white noise added to the signal, since Basu gives a model for edge enhancement and not for edge detection.

\section{Degraded edge-object and edge-image intensity distributions}

We consider an image forming system, working under incoherent illumination, and having a circular pupil of unit radius (see Fig. 1). Let $\left(x_{0}, y_{0}\right)$ be the rectangular coordinates at the object plane. In this plane we define the degraded edge as:

$D E_{0}\left(x_{0}\right)=H\left(x_{0}\right)\left[1-\exp \left(\frac{-x_{0}}{\tau}\right)\right]$

where $\tau$ is the degradation parameter (with dimensions of a length) and $H\left(x_{0}\right)$ is the Heaviside or step function. The term in brackets represents the degrading factor. These edges are non-symmetrical: they do not present a curve zone in the transition from zero intensity to the increasing values when the spatial variable $(x)$ increases (see Fig. 2). Since there is a

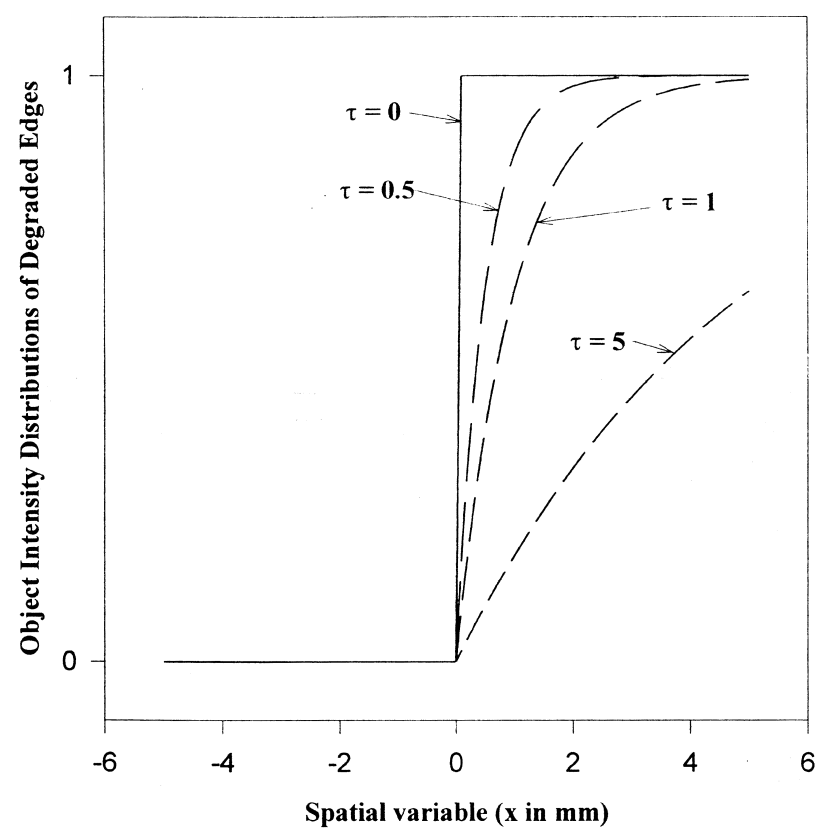

Fig. 2. Intensity distribution associated with the edge object as defined in Eq. (1). To account the effect of the degradation parameter several values of $\tau$ are considered. The gray level is 0 for white and 1 for black. 
sudden change in the intensity, they do not fit with a sigmoig as many authors suppose, ${ }^{*}$ but clearly saturation is not actually a symmetric effect, only the function appears to be mathematically more manageable. Fig. 2 shows the shape of the degraded edge-object for some values of $\tau$. The parameter $\tau$ is related to the smoothness of the edge-object, thus $\tau=0$ reproduces the perfect edge, while $\tau \gg 1$ accounts for a highly degraded one. The degradation parameter represents approximately the half-distance required for the step to increase from 0 to $90 \%$ its maximum intensity (see Fig. 3). It can be seen as the blur width [1] of the degraded edge. Eq. (1) is defined as a continuous function (with continuous derivatives) that will guarantee a suitable numerical behavior.

In the Fourier plane, with coordinates $(u, v)$, the object Fourier transform $F(u, v)$ of Eq. (1) is filtered by the optical transfer function $(O T F)$ of the system. For simplicity the pupil of the system is located close to the Fourier transform plane (a negligible distance to it). This simplification implies that we consider a telecentric effective stop [20] and introduces the diffraction limited conditions of the system. The filtered Fourier transform signal is:

$F D E(u . v)=F(u, v) \cdot O T F(u, v)$

where,

$$
\begin{aligned}
F(u, v)= & \tau \delta(\nu)\left[\frac{\delta(u)}{2 \tau}-\frac{1}{1+(2 \pi \tau u)^{2}}\right. \\
& \left.-i\left[\frac{(2 \pi \tau u)}{1+(2 \pi \tau u)^{2}}-\frac{1}{2 \pi \tau u)}\right]\right]
\end{aligned}
$$

$\operatorname{OTF}(u, v)=\operatorname{circ}\left(\frac{r}{2}\right) * * \operatorname{circ}\left(\frac{r}{2}\right) \operatorname{circ} * * \operatorname{circ}$

and $* *$ denotes autocorrelation, $\delta$ is the Dirac-delta function, and $\operatorname{circ}(r / 2)=1$ (for $\left.r=\sqrt{ }\left(u^{2}+v^{2}\right)<1\right), 0$ (for $r>1$ ).

The image intensity distribution at the image plane, $D E(x$, $y$ ), is the inverse Fourier transform of $F D E(u, v)$ given in Eq. (2). As $F(u, v)$ depends on a single coordinate, its inverse Fourier transform also depends on a single one:

$D E(x)=D E_{0}(x) *\left(\frac{J_{1}(2 \pi \rho)}{\rho}\right)^{2}$

with $D E_{0}(x)$ as expressed in Eq. (1), $\rho=\sqrt{ }\left(x^{2}+y^{2}\right)$ is the radial coordinate, and $*$ denotes correlation. Then,

$D E(x)=\int_{-\infty}^{+\infty} D E_{0}(x-\alpha)\left\{\int_{-\infty}^{+\infty}\left[\frac{J_{1}\left(2 \pi \sqrt{\left(\alpha^{2}+\beta^{2}\right)}\right)}{\sqrt{\left(\alpha^{2}+\beta^{2}\right)}}\right]^{2} \mathrm{~d} \beta\right\} \mathrm{d} \alpha$

The expression between parentheses is the well-known $L S F$ of a perfect system having a circular pupil of unit radius, denoted by $\operatorname{LSF}(\alpha)$. It follows:

$D E(x)=\int_{-\infty}^{+\infty} D E_{0}(x-\alpha) L S F(\alpha) \mathrm{d} \alpha$

\footnotetext{
* For example, Shanmugan et al. in [1] introduced the sigmoid function as a symmetric function to represent an edge affected by a gray level as a degradation from the capturing procedure of the detector.
}

and, according to Eq. (1),

$D E(x)=\int_{-\infty}^{+\infty} L S F(\alpha) \mathrm{d} \alpha-\exp \left(\frac{-x}{\tau}\right) \int_{-\infty}^{x}\left(\frac{\alpha}{\tau}\right) L S F(\alpha) \mathrm{d} \alpha$

Eq. (8) is the key equation to represent the image intensity distribution of a degraded edge. To obtain an analytical expression for Eq. (8) one needs to substitute the $L S F$ for a more straightforward expression which can be easy to compute. A certain number of exact formulas and approximations are given in the literature [21,22] but we use here the expression recently proposed by us [23]:

$$
\begin{aligned}
\operatorname{LSF}(\alpha)= & \int_{-\infty}^{+\infty}\left[\frac{J_{1}\left(2 \pi \sqrt{\left(\alpha^{2}+\beta^{2}\right)}\right)}{\sqrt{\left(\alpha^{2}+\beta^{2}\right)}}\right]^{2} \\
& \times \mathrm{d} \beta=\frac{8}{\pi^{2}} \sum_{n=1}^{\infty} \frac{n^{2}}{4 n^{2}-1} \frac{J_{2 n}(4 \pi \alpha)}{\alpha^{2}}
\end{aligned}
$$

where $J_{2 n}(x)$ is the Bessel function of order $2 n$. By substituting Eq. (9) into Eq. (7), the degraded edge image intensity distribution is:

$$
\begin{aligned}
D E(x)= & \frac{16}{\pi^{3}} \sum_{n=1}^{\infty} \frac{n^{2}}{4 n^{2}-1} \int_{-\infty}^{x} \\
& \times\left[1-\exp \left(\frac{-x+\alpha}{\tau}\right)\right] \frac{J_{2 n}(4 \pi \alpha)}{\alpha^{2}} \mathrm{~d} \alpha
\end{aligned}
$$

The final result (Eq. (10)) has been normalized in this notation to:

$D E(\infty)=\int_{-\infty}^{+\infty} L S F(\alpha) \mathrm{d} \alpha=\frac{\pi}{2}$

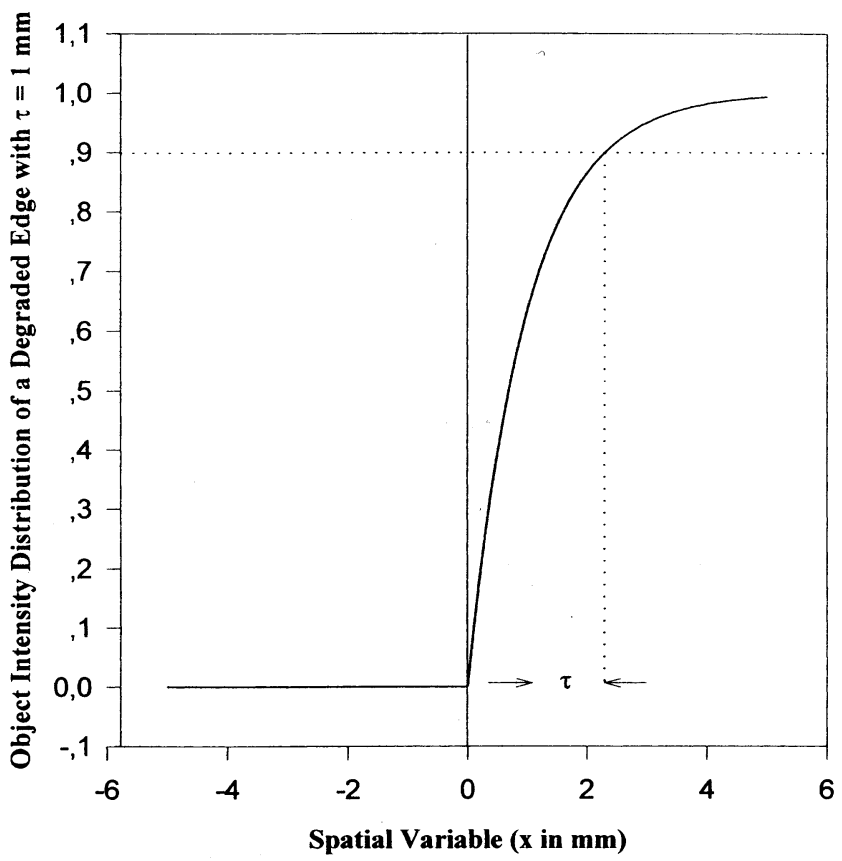

Fig. 3. Graphical explanation of the meaning of a degradation parameter $(\tau)$. 
Eq. (10) represents the ESF for blurred edges. The possible oscillations arising from the numerical behavior of the Bessel functions $[24,25]$ are suppressed due to the optimized Bessel functions expansion used in the computation [23]. As an example, Fig. 9(a) shows the comparison between the theoretical and experimental image intensity distribution for a focused edge processed in a real optical system. Only oscillations due to experimental procedure will appear. Obvious differences appear comparing the theoretical object and image distributions. The transition from zero intensity to higher intensity is smoother in the imaged one: a gradual change in the slope appears in this zone for edges with low degradation parameters $(0<\tau<1)$. Obviously, this feature disappears for higher defocused edges $(\tau \gg 1)$. Thus, the geometrical shadow region of the edge is not equally defined. As the argument reaches zero, low degraded edges will cut the intensity axis at a particular point, having a well-defined geometrical shadow (for example, for data not displayed here for brevity, the area under the left elbow on the image intensity distribution, the intensity varies from $65 \%$ to $95 \%$ ).

\section{LSF and MTF associated with degraded edges}

One can apply the well-known operation for the $L S F$ to be obtained by differentiating the $E S F$, or inversely, the $E S F$ is calculated by integrating the line response or $L S F$ in the orthogonal direction to its axis [14-16]. Thus, we define the one dimensional gradient of the edge image intensity distribution as an $L S F$ associated with this specific edge. The gradient of the incoherent edge intensity distribution

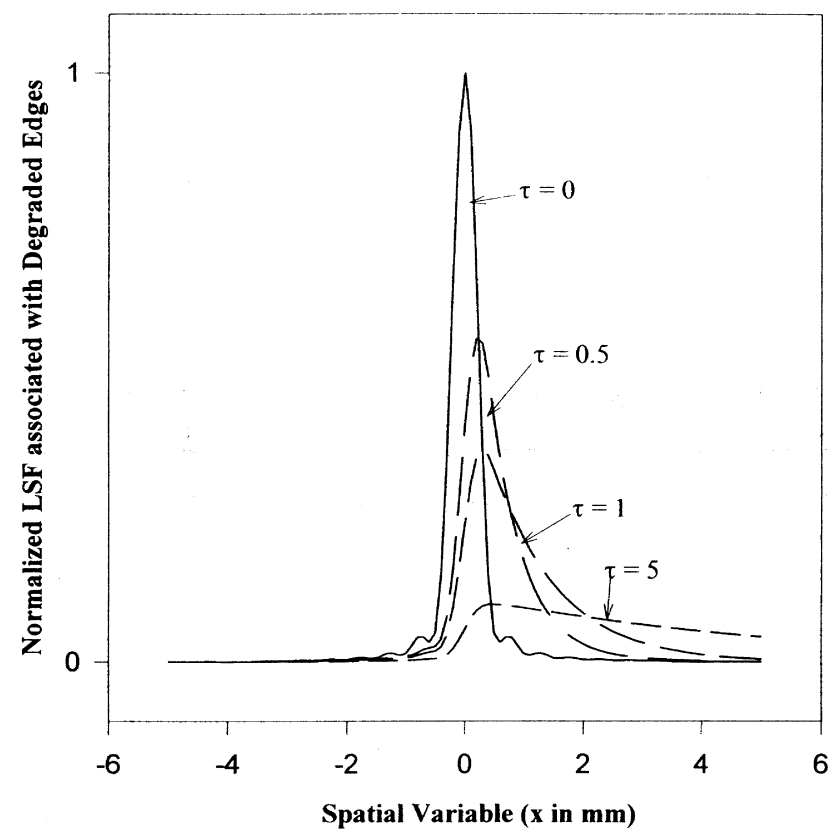

Fig. 4. LSF associated with the image of the degraded edge objects, according to Eq. (15). The ' $X$ ' represents the spatial coordinate. Ordinate axis represents the line radiant flux density (in arbitrary units). decreases uniformly and continuously across the spatial coordinate and it is a measure of the image quality [14] in the case of simple defocusing. Since edge degradation can be a consequence of defocusing, this associated $L S F$ gives information on the edge image quality:

$L S F_{\mathrm{d}}(x)=\frac{\mathrm{d}}{\mathrm{d} x}(D E(x))$

By using the Leibniz rule [24] to differentiate under the integral sign in Eq. (12):

$L_{S} F_{\mathrm{d}}(x)=\frac{16}{\pi^{3} \tau} \sum_{n=1}^{\infty} \frac{n^{2}}{4 n^{2}-1} \int_{-\infty}^{x} \exp \left(\frac{-x+\alpha}{\tau}\right) \frac{J_{2 n}(4 \pi \alpha)}{\alpha^{2}} \mathrm{~d} \alpha$

Eq. (13) is represented in Fig. 4 for various degradation parameters $(0 \leq \tau \leq 5)$. For $\tau>0$ the shape of this $L S F_{\mathrm{d}}$ is not a symmetrical one. This feature appears directly connected to the definition of the real degraded edges, that are inherently non-symmetrical. Moreover, the higher the degradation parameter, the more spreading the $L S F_{\mathrm{d}}$. As the degree of blur increases, the energy distribution tends to equalize over the spatial coordinate and the central maximum decreases. This corresponds to an edge image highly degraded and embedded into the geometrical shadow, as commented in the later section. Opposite, if the degradation parameter is smaller than unity $(\tau<1)$, the $L S F_{\mathrm{d}}$ shows a high central peak: the distribution of the energy is concentrate at the origin (location of the edge).

We shall introduce a new definition: the modulation transfer function associated with a degraded edge $\left(M T F_{\mathrm{d}}\right)$. This magnitude provides information on the response of the system to degraded edges for a spatial frequency range, giving data on the contrast. The cut-off frequency remains constant for all defocused edge objects [26], as it is determined by the system aperture, but the shape of the $M T F_{\mathrm{d}}$ will vary according to the degradation parameters. As is well-known [27,28], the MTF of a rotationally invariant system, whose $P S F$ depends on a single variable, is given as the modulus of the Fourier transform of the $L S F_{\mathrm{d}}$. Therefore,

$\operatorname{MTF}_{\mathrm{d}}(u)=\left|F T\left(L S F_{\mathrm{d}}(x)\right)\right|$

where $u$ is the spatial frequency of the system. By using a well-known property of the Fourier transform related to the derivative:

$\operatorname{MTF}_{\mathrm{d}}(u)=\left|F T\left[\frac{\mathrm{d}}{\mathrm{d} x} D E(x)\right]\right|=|2 \pi i u F T(D E(x))|$

Notice that this theorem has restrictions: it must be applied to monotonically decreasing functions $D E(x)$. Our case satisfies this condition, since we are considering a diffraction-limited system (limited by the aperture pupil) where the edge function is zero outside the pupil region. Application of derivatives enhances the high frequencies, attenuates the low ones and suppresses the zero frequency, as the operation 
corresponds to a filtering process with a complex filter with constant phase shift $\pi / 2$ and linear attenuation.

By considering that the edge image intensity distribution is given by the convolution between the edge object and the $L S F$ of the system, the Fourier transform appearing in the right-hand-side of Eq. (15) is:

$$
\begin{aligned}
& F T[D E(x)]=F T\left[D E_{0}(x) * \operatorname{LSF}(x)\right] \\
&=F T\left[D E_{0}(x)\right] F T[\operatorname{LSF}(x)] \\
&=F T\left[D E_{0}(x)\right] \operatorname{MTF}(u) \\
&=\frac{16}{\pi^{3}} \sum_{n=1}^{\infty} \frac{n^{2}}{4 n^{2}-1} \int_{-\infty}^{+\infty} F T\left[\left(1-\exp \left(\frac{-x+\alpha}{\tau}\right)\right) H(x-\alpha)\right] \\
& \quad \times F T\left[\frac{J_{2 n}(4 \pi \alpha)}{\alpha^{2}}\right] \mathrm{d} \alpha
\end{aligned}
$$

Eqs. (15) and (16) denote that the $M T F_{\mathrm{d}}(u)$ is proportional (factor $2 \pi i u$ ) to the product of the Fourier transform of the degraded edge-object and the MTF associated with the perfect optical system forming the image.

By calculating the two FT [25] of Eq. (17) and substituting the result, we find:

$\operatorname{MTF}_{\mathrm{d}}(u)=\mid \frac{32 i}{\pi} u \sum_{n=1}^{\infty} \frac{8 n^{2}}{4 n^{2}-1} \cdot{ }_{2} F_{1}\left[-n-\frac{1}{2}, n-\frac{1}{2}, \frac{1}{2} ; \frac{u^{2}}{4}\right]$.

$$
\left(\frac{\delta(u)}{2}-\frac{\tau}{1+(2 \pi u \tau)^{2}}-\tau i\left[\frac{2 \pi i \tau u}{1+(2 \pi \tau u)^{2}}-\frac{1}{(2 \pi \tau u)}\right]\right)
$$

where ${ }_{2} F_{1}[a, b, c ; z]$ is the Hypergeometric function of first kind and second order [28,29]. This result is valid for $0<u$

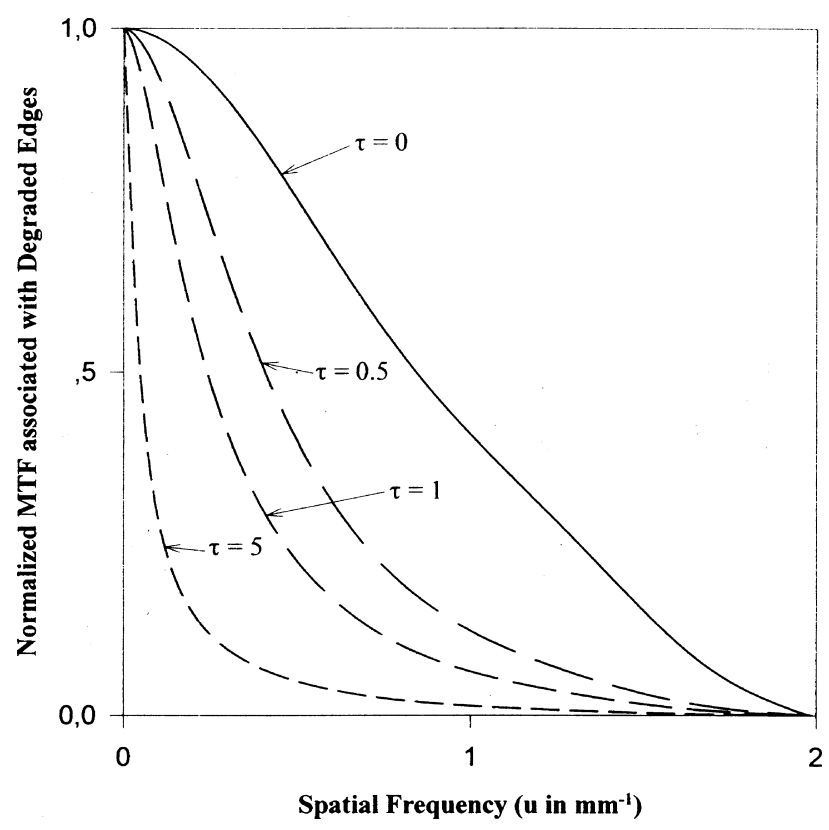

Fig. 5. MTF associated with the image of degraded edge objects, according to Eq. (20).
$<2$, that represents the cut in frequencies due to the pupil size limit. Fig. 5 shows the shape of the $M T F_{\mathrm{d}}$ for some values of the degradation parameter. For $0<\tau<1$, the associated $M T F_{\mathrm{d}}$ reduces monotonically its contrast, while for $\tau \gg 1$ very highly degraded edges exhibit a similar fast decreasing shape.

This feature may play an important role in edge recognition. Obviously, matched filtering adapted to a specific edge object with a certain degradation (a specific $\tau$ ) will be required, but to recognize a large class of highly degraded edges having different degree of blur (whenever the $\tau$ value was high), a unique correlation will operate.

\section{Contrast reduction function}

According to Eqs. (15) and (16), the $M T F_{\mathrm{d}}$ depends on the $M T F$ of the perfect system. By using the properties of the modulus, we have:

$\operatorname{MTF}_{\mathrm{d}}(u)=|2 \pi i u F(u) M T F(u)| \leq|2 \pi i u F(u)| \cdot|\operatorname{MTF}(u)|$

where $F(u)$ is given in Eq. (3). The $\operatorname{MTF}(u)$ can be calculated by taking the modulus of the Fourier transform of the perfect system's $L S F$ (see Eq. (9)). Here, one can define the ratio $M T F_{\mathrm{d}}(u) /|M T F(u)|$ where the contrast reduction be related to the edge-object quality. Thus:

$C_{\mathrm{d}}(u)=\frac{M T F_{\mathrm{d}}(u)}{|M T F(u)|} \leq|2 \pi i u F(u)|$

The degradation mechanism appears to be bounded by the nature of the Fourier transform of the edge-object. By substituting Eq. (3) in Eq. (20) and operating, one obtains:

$C_{\mathrm{d}}(u) \leq \sqrt{\frac{1}{1+(2 \pi \tau u)^{2}}}$

As a proof of consistence, one evaluates the value of the contrast for the zero-frequency component. This value has to be equal to the unity. It is immediate to realize that: $C_{\mathrm{d}}(0)$ $\leq 1$. On the other hand, for $\tau=0$ we obtain the perfect edge contrast, that is, the contrast without reduction since $C_{\mathrm{d}}(\mathrm{u}, \tau$ $=0)=1$.

Fig. 6 displays the contrast reduction function for $0 \leq \tau \leq$ 5. As an example, for $u=1 \mathrm{~mm}^{-1}$ one has that from $0 \leq \tau$ $\leq 0.5$ the reduction is about $70 \%$, but from $0 \leq \tau \leq 1$ is $85 \%$. For $\tau=5$ the contrast is very low since the contrast reduction is about $97 \%$. Fig. 6 shows that the contrast reduction depends on edge-object degradation: the higher the degradation parameter, the larger the contrast reduction in relation to the hard perfect edge. For very low spatial frequencies $(0 \leq u \leq 0.05)$, the contrast reduction for $0 \leq \tau \leq$ 5 is small, since all shapes are over the $50 \%$ of contrast. For low spatial frequencies $(0.05 \leq u \leq 0.2)$, the contrast for 0 $\leq \tau \leq 1$ decreases but not dramatically. However, for $\tau>1$ the contrast is under 0.5 . For an intermediate range of spatial frequencies $(0.2 \leq u \leq 1)$ the contrast diminishes strongly 


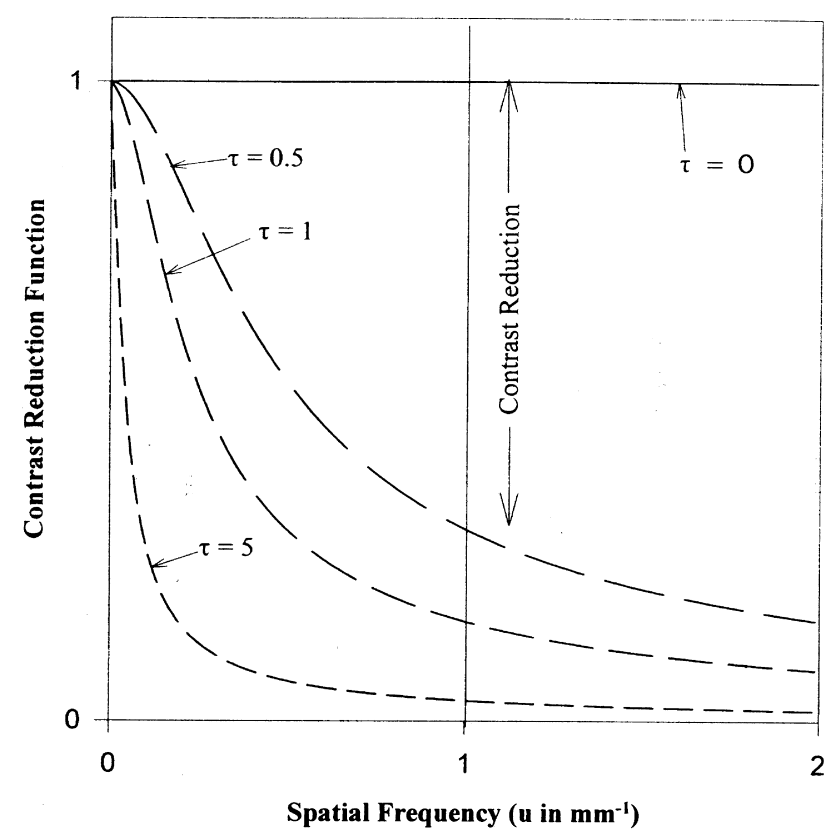

Fig. 6. Contrast reduction function, according to Eq. (21). Two behaviors can be determined: (a) $0 \leq \tau \leq 1$, the contrast function decreases monotonically (there is a contrast reduction for high frequencies); (b) $1<\tau \leq 10$, the shape is rather similar for all $\tau$ (there is a dramatic reduction for high frequencies).

for all $\tau$. The shapes for $\tau>1$ become similar and tend to equalize as the spatial frequencies increase. For very high values of the spatial frequency $(u \geq 1)$ and degradation parameters of $0 \leq \tau \leq 1$, the contrast reduction tends to the zero value, although for higher $\tau$ it tends faster. As the spatial frequency increases, the contrast also diminishes. Related for example to visual acuity, a reduction on the contrast for higher spatial frequencies has been reported for defocused small letter contrast sensitivity (SLCS) [30]. The results show that a shift takes place with respect to the contrast sensitivity for focused SLCS. The change in the slope of the contrast sensitivity function, depending on the object contrast, implies a reduction in visual acuity.

On the other hand, Artal and Navarro have obtained the monochromatic MTF of the human eye for different pupil diameters [31], where a similar behavior for degradation to the one we are discussing is presented. The MTFs are fitted by using an analytical expression in terms of a two exponential functions addition: the function with higher weight and slope fits the low-spatial frequency range, and the other fits the high spatial frequency range. In this case, no cut-off frequency can be defined since the exponentials never reach zero and, therefore, these parametric $M T F$ s yield $P S F$ s that are narrower than the actual ones.

\section{Experimental degraded edges}

In order to prove the correctness of our representation for real degraded edges, we have set-up an experimental system displayed in Fig. 7. The system carries out all the requirements expressed in Section 2 related to degraded edges. Edge degradation is taken by defocusing the optical system.

We used the monochromatic illumination from a heliumneon/20 mW laser. Since we needed temporal incoherent light (to suppress all correlations from temporal and spatial interferences), a spatial filtering operation (SPF) is made up and a rotating diffuser (RD) is placed. A condenser lens (CL) is located after the diffuser (where the incoherent source is now defined, having a certain extension or small area) to collect the light and to give a uniform illumination at the object plane $\left(D E_{0}\left(x_{0}, y_{0}\right)\right)$ where a knife edge (halfplane) acts as a perfect straight edge. The object plane is located at a distance $d_{\mathrm{o}}$ from a convergent lens (L), with focal distance $f=10 \mathrm{~cm}$, that forms the image intensity distribution at the image plane, at a distance $d_{\mathrm{i}}$. Both

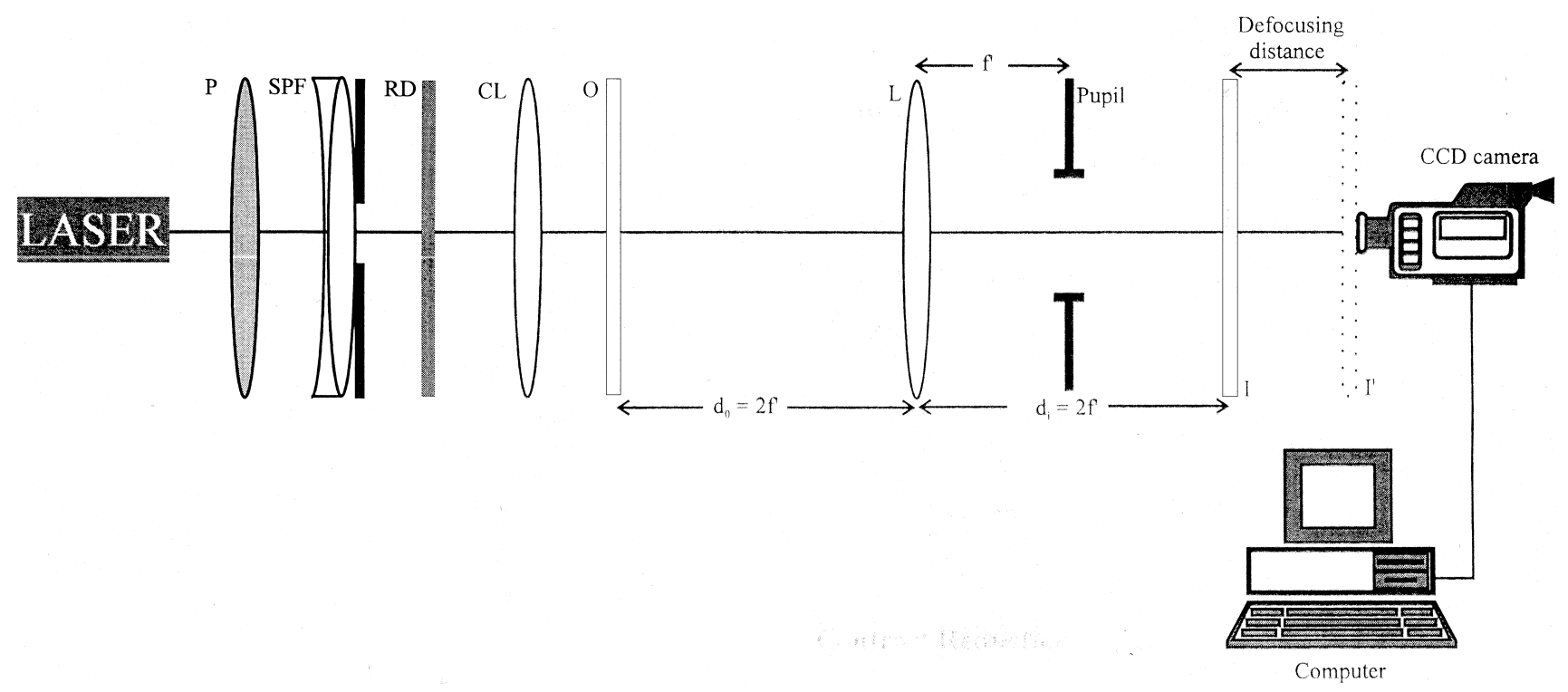

Fig. 7. Experimental optical system (see text for details). 
distances $\left(d_{\mathrm{o}}\right.$ and $\left.d_{\mathrm{i}}\right)$ are approximately related by the conjugation relation for convergent thin lenses. In the image plane, a CCD camera connected to a PC computer is placed. We have used a Data Translation 2861 frame grabber to capture the images from the CCD camera to the computer. In order to avoid excessive saturation of the camera, a polarizer $(\mathrm{P})$ is placed after the laser. By rotating the polarizer, one can select the amount of illumination in the system. To obtain the defocused or degraded edges, the image plane I is shifted to some different positions I' far from the distance $d_{\mathrm{i}}$ (the position of perfect focusing or perfect straight edge). This positive and increasing distance is denoted by the defocusing distance (denoted in $\mathrm{mm}$ ).

Fig. 8(a)-(e) displays various photographs of degraded edges, for defocusing distances: 0 (focused edge), 2, 5, 10 and $20 \mathrm{~cm}$; and Fig. 8(f)-(j) shows their respective line profiles. The shape for the edge-image intensity distribution associated with various experimental blurred edges fits apparently with the theoretical predictions, as can be seen in Fig. 8(f)-(j). For small defocusing distances $(0,2$ and $5 \mathrm{~cm})$, the slope of the edge is higher. Defocusing causes an increasing shift with respect to the location of the hard edge. The slope for high defocused edges becomes lesser than for the hard perfect edge, since the degree of gray levels increases.

Apart from the localization of the edge, which is given by the argument of the Heaviside function, two main characteristics arise from the comparison between the theoretical and the experimental edges (Fig. 9(a)): intensity degradation and noise background. It is necessary to consider this features to fit exactly our model with the experimental results.

\subsection{Intensity degradation}

The decreasing of the maximum intensity is due to the way we use to degrade the original hard edge. Since we remove the image plane far from its focusing position, less light will be captured by the CCD detector each time and, therefore, less intensity will arrive. This characteristic can be observed in Fig. 8(f)-(j) where the edge intensity decreases in proportion to the increasing of defocus. In Fig. 8(g) it is displayed the quantity 'edge height' $(E H)$, that represents mathematically the intensity diminish.

\subsection{Noise background}

In Fig. 8(h) one can see two types of noise. Electronic noise appears in every experimental set up. It is present in the small oscillations of the shape and it is caused by the recording device (CCD camera). It only supposes a $1 \%$ in intensity oscillations. Noise background appears in the zone of the edge that should have zero values of intensity (black zone of the edge). This happens because the hard edgeobject used to generate the object was not totally opaque and then light could pass through the black part of the edge giving a background, which was not considered in the model. We call this background noise 'edge opacity' (EO) and it is approximately equal for all the imaged edges, since the object edge is physically the same for all.

We introduce this two parameters, as well as the edge location, by simply operating in the previous definition of the degraded edge (Eq. (1)):

$$
D E_{0}\left(x_{0}\right)=E O+E H \cdot H\left(x_{0}-a\right)\left(1-\exp \left(\frac{-x_{0}+a}{\tau}\right)\right)
$$

with $a=$ localization of the edge and the restrictions $0 \leq E O$ $\leq 1$ and $0 \leq E H \leq 1$.

Convolving this expression with the $L S F$ of the perfect system (Eq. (9)), one obtains the theoretical fitting to the empirical edges. Fig. 9(a) displays the comparison between the theoretical, experimental and fitted shape for the focused edge. Fig. 9(b) shows the fitting and the experimental shapes for the five captured edges. The model fits better with the low degraded edges than with the high degraded ones, since the low degraded edges have a well-defined geometrical shadow and therefore they are easily reconstructed by the system. For high degraded edges, the image intensity distribution does not present the same behavior since the gradual change of the slope is not well-defined. This gives rise to an ambiguous geometrical shadow region (intensity varying from $10 \%$ to $20 \%$ ) and avoids a correct definition of it. In this case, the degraded edge is embedded into the geometrical shadow and it is impossible to reconstruct or recognize it, due to the small variation in the slope, and therefore, a dramatic reduction of contrast takes place (see Fig. 9(b)).

Starting from this curve fitting the empirical edges, one can derive the already defined associated functions ( $L S F$, $M T F, C R F$ ) and obtain information about image quality, frequencies processed and contrast reduction by applying the previous formalism. Fig. 10 displays the $M T F$ associated with the experimental edges given by the line profiles of Fig. 8(f)-(j). We have represented also the MTF of the theoretical focused edge. The experimental focused edge appears degraded in comparison with the theoretical one, but not excessively since the merit function (area between the theoretical prediction and the experimental result) is negligible. As it is expected, when defocusing increases, the modulation transfer decreases. Fig. 11 shows the associated $C R F$. This function has been calculated by dividing the $M T F$ associated with an experimental edge of a certain degradation and the MTF associated with the theoretical focused edge. This fact is somehow a normalization that gives the contrast reduction of the experimental imaging related to the theoretical predictions. Although the $C R F$ is mathematically given in terms of the edge object spectrum, it is not possible experimentally to apply this relation, since we do not know a priori the values of the parameters $E O, E H$ and $\tau$. Therefore, $C R F$ is given experimentally as a rate of both MTFs. 
Another interesting magnitude can be computed: the depth of focus (DOF) of the system. There are several ways to define the DOF:

- The dioptric distance between the two points that determine the depth of field of the system [26].

- The dioptric range for which the $M T F$ exceeds the $50 \%$ of its maximum for a given spatial frequency [26].

- The range of defocus for which the $C R F$ exceeds the $80 \%$ of its maximum for a given frequency [32].

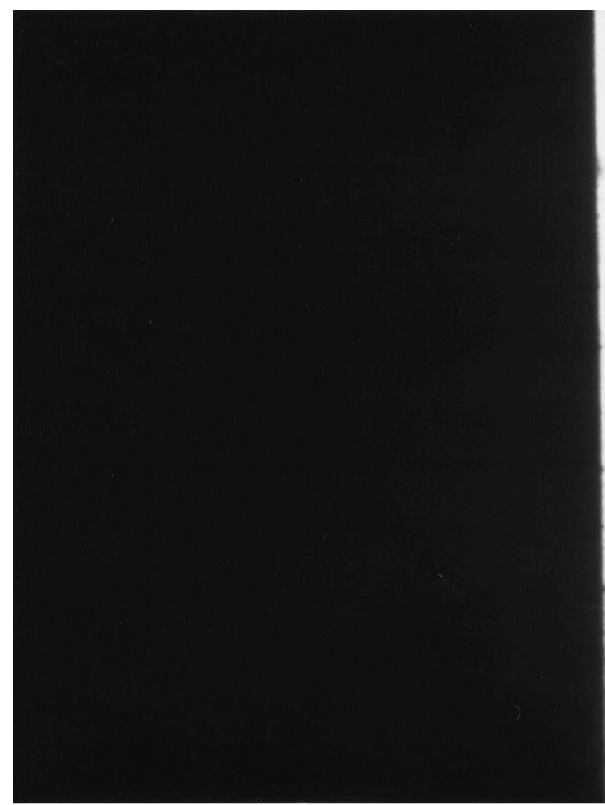

(a)

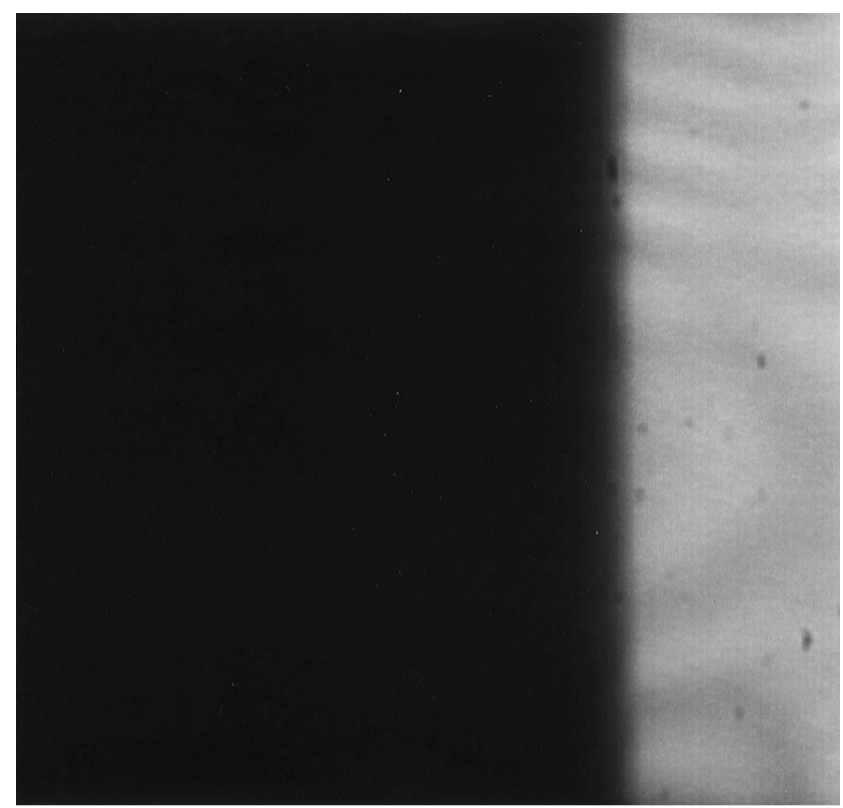

(c)
In any case it is possible to give a value for the DOF starting from other known functions. In this work, we use the $C R F$ to compute the DOF in terms of defocusing for an average spatial frequency. The election of this frequency depends on the considered experimental system. We show a practical case of the DOF fixing a spatial frequency value and representing the $C R F$ in terms of the defocusing $(\tau)$. The range of defocusing for which the $C R F$ surpass $80 \%$ of its maximum value is a measure of the depth of focus, which is given in

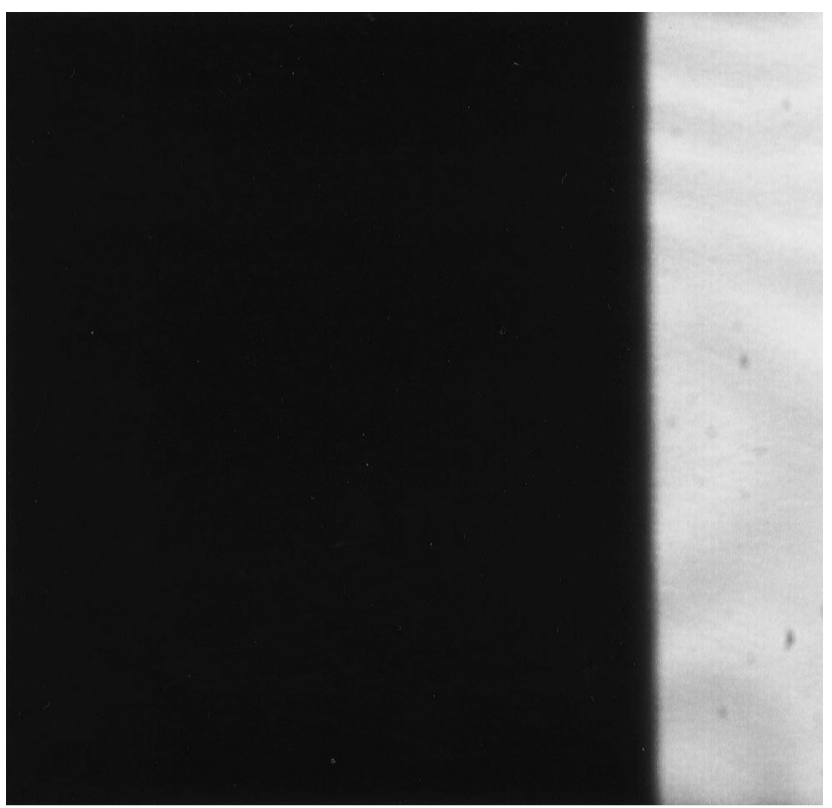

(b)

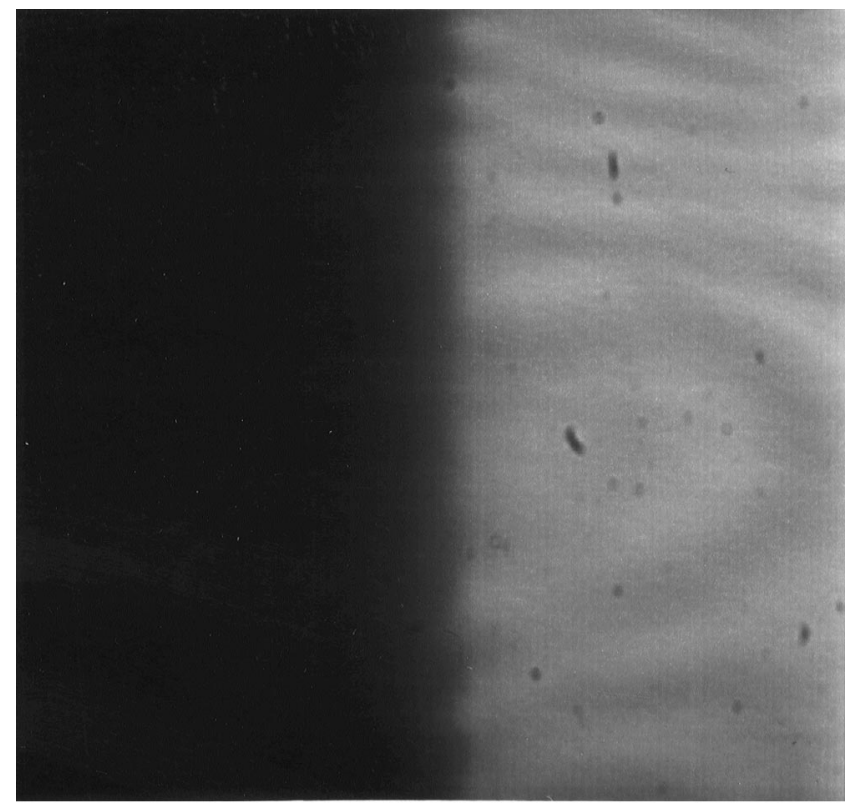

(d)

Fig. 8. Images captured of the experimental degraded edges, for various defocusing distances: (a) focused edge $(0 \mathrm{~cm})$; (b) defocusing $=2 \mathrm{~cm}$, (c) defocusing $=5 \mathrm{~cm}$; (d) defocusing $=10 \mathrm{~cm}$ and (e) defocusing $=20 \mathrm{~cm}$. The corresponding line profiles of these images are given in (f)-(j). All the line profiles were centered at the 256 pixel line of the digitized image $(512 \times 512)$ and normalized with respect the maximum gray level of the hard edge $(255=$ absolute white). The ' $X$ ' represents the spatial coordinate. Since the optical image of the degraded edge has been digitized, the spatial coordinate was translated from pixels to millimeters, using the conversion: 1 pixel $=0.013 \mathrm{~mm}$. The gray level is 1 for white and 0 for black. 
dioptric units since $\tau$ has units $\mathrm{mm}^{-1}$. Then, we can obtain an objective measure of the system depth of focus solely having the image of some defocused edges processed by the system, as well as other information of interest. Fig. 12 shows the results for the $C R F$ in terms of defocusing. In this case, for $u=1$, the depth of focus is $1.24 \mathrm{~mm}^{-1}$ (diopters).

\section{Discussion and conclusions}

We have presented in this paper a characterization of degraded edge imaged by a perfect system by introducing a convenient 'degradation parameter' $(\tau)$. This parameter is

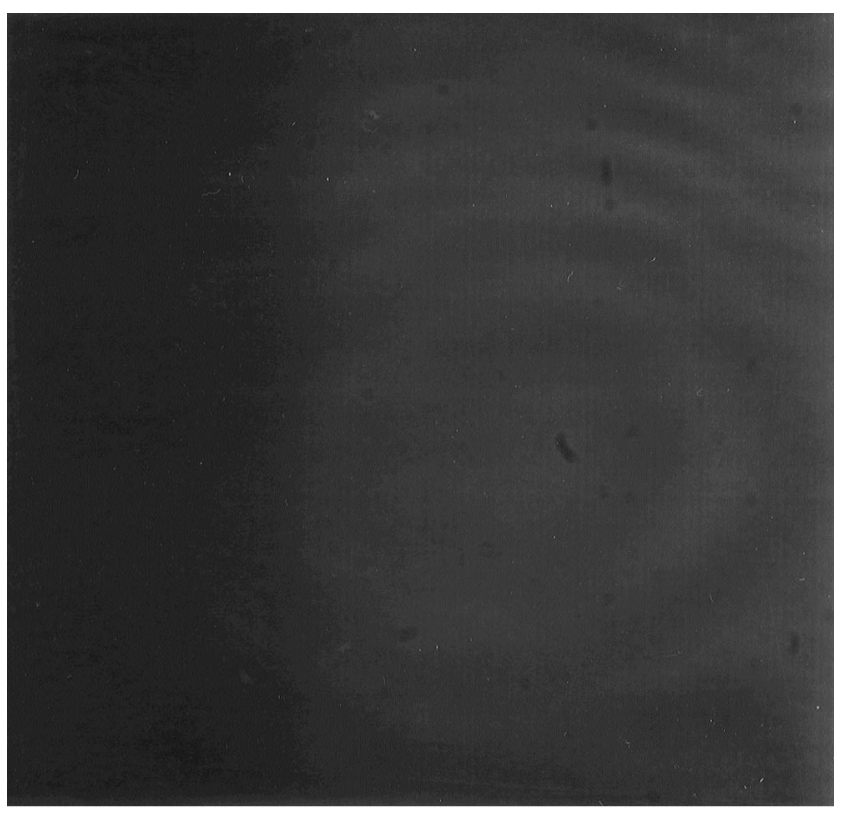

(e)

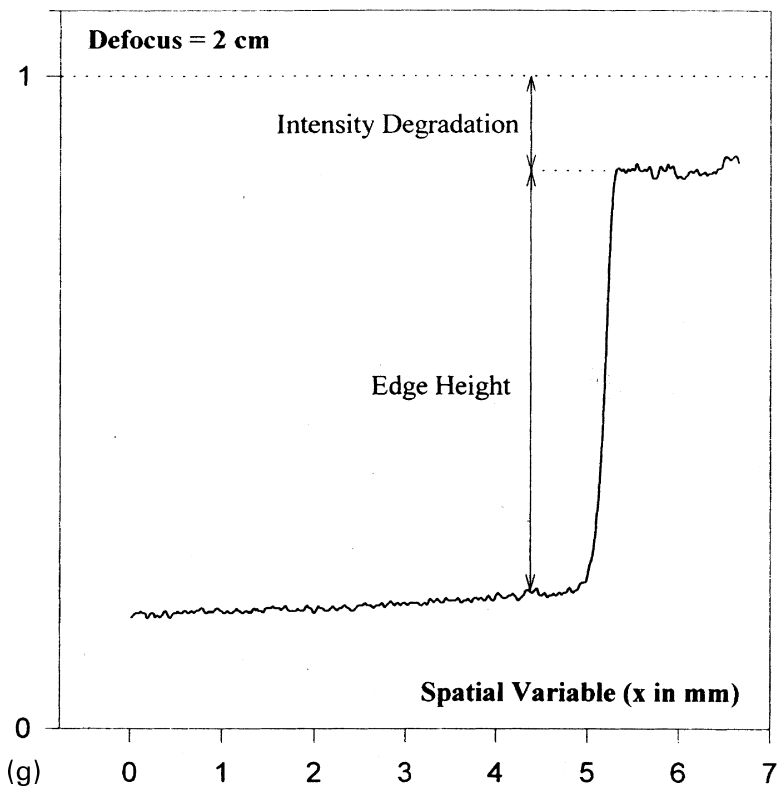

related to a defocusing in the image plane in any experimental diffraction limited system with circular pupil under conditions of incoherent illumination. To typify a degraded edge it is necessary and useful for many applications to quantize the degree of blur. For example, a scene (e.g. a real life picture) is basically composed of many objects all defined within certain boundaries with their corresponding edges (or gradual changes in the luminance). Depending on the depth situation of the object in the visual scene, the edges of the boundaries become more or less degraded. According to Pentland's psychophysical experiments [18], the further the object, the more degraded are its boundary edges. Observers can interpret an increase in defocusing as
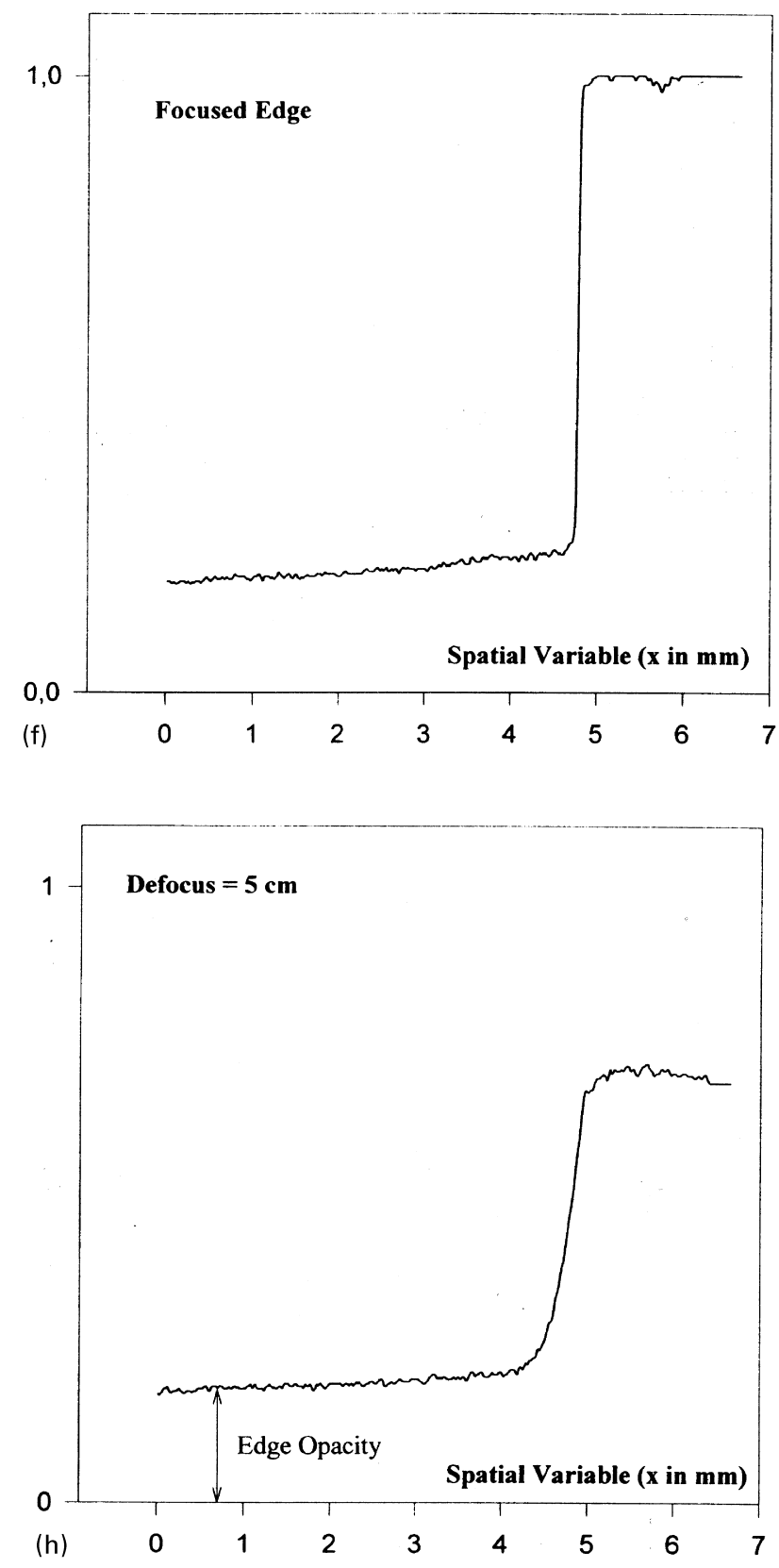

Fig. 8. Continued 

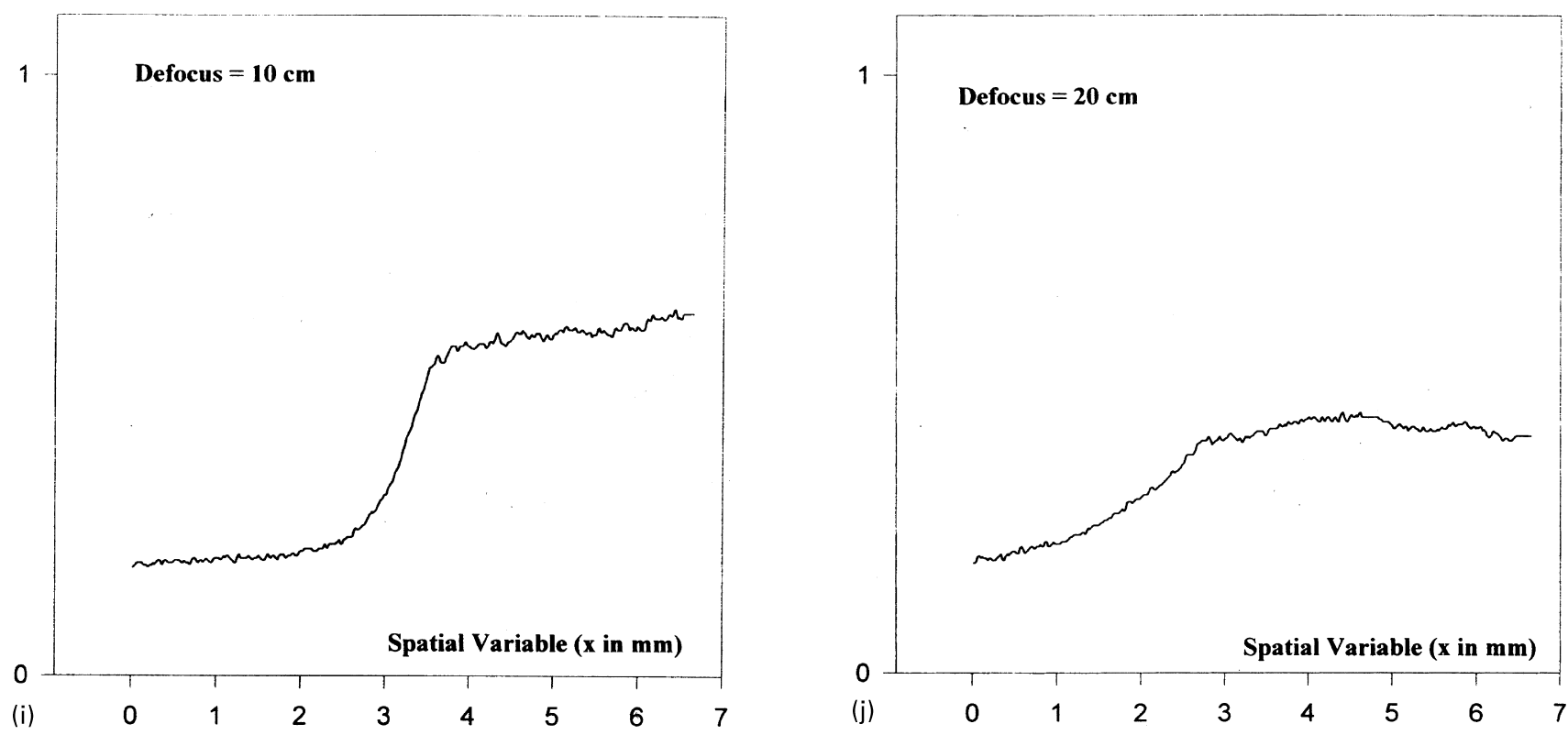

Fig. 8. Continued

an increase in distance, maybe because they focus having as a reference the nearest object in the visual field. In this sense, it is interesting to characterize the degraded edges of the scene, not as a function related to the imaging system (as a degraded $L S F$ ), but as a function that describes the edges themselves, defining intrinsic characteristics, and the degraded edge as a depth cue. These avoid connecting the degrading mechanism to the concrete features of the system that provides the scene.

To study a method to characterize the degraded edge, we first analyze the associated image intensity distribution. Its expression is given in Eq. (12) in terms of a convolution between the degraded edge object and the $L S F$ associated with a perfect system. In the interval
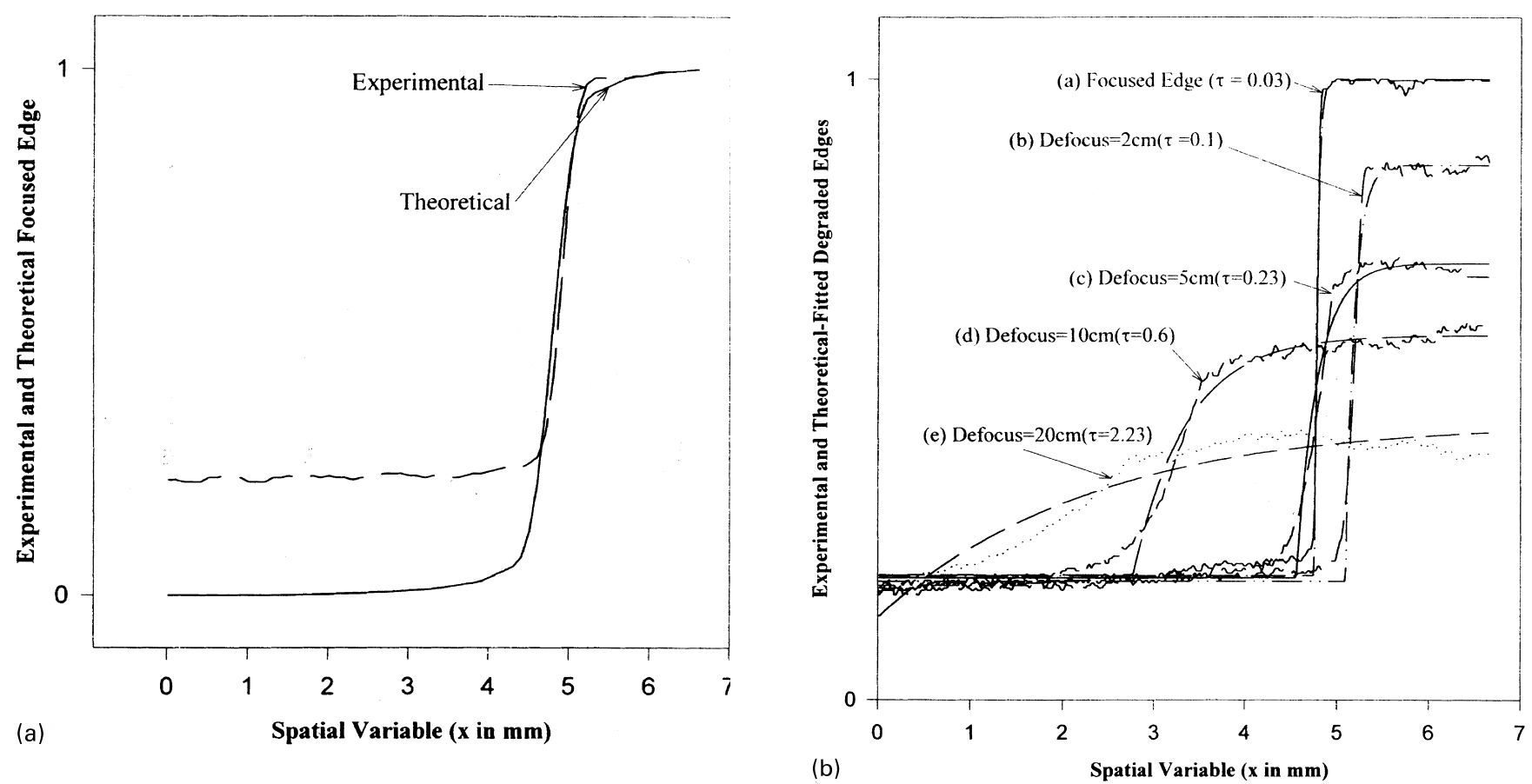

Fig. 9. (a) A joint representation of the experimental (dashed line) and theoretical (solid line) intensity image distribution for the focused edge. (b) Experimental degraded edge images as in Fig. 8(f)-(j) fitted with the modified formula (see Eq. (22)) for the corresponding (values (defocusing equivalence). For focused and low defocused edges $(0-5 \mathrm{~cm})$ the model fits better, apart from experimental electronic noise. The edge opacity remains unchanged for all the considered cases. The edge heights are related with the slope. For high defocused edges the standard deviations are important. 


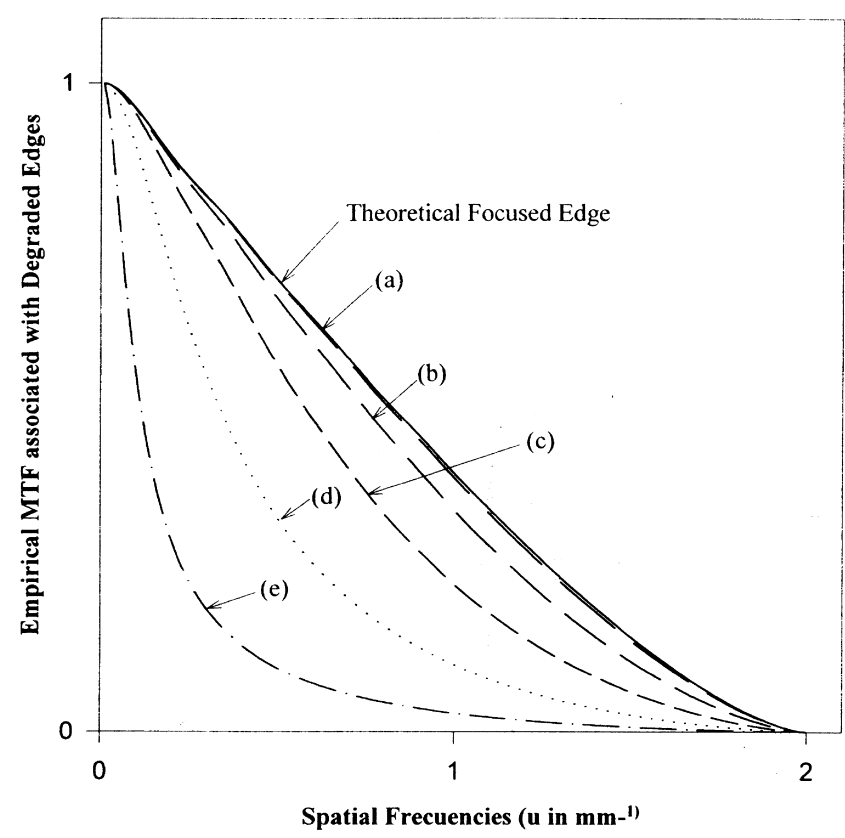

Fig. 10. Empirical MTF obtained from the experimental fitted edge images given in Eq. (9). The perfect and the focused have a similar MTF. As $\tau$ increases (increasing defocusing) the MTF behaves as a narrower low-pass filter.

under analysis (see Fig. 4), the degraded edges have a particular stabilization for increasing arguments: they tend to unity, but as the degradation parameter increases, the tendency is less noticeable. Two main magnitudes characterize the edge-image intensity distribution: edge oscillations and

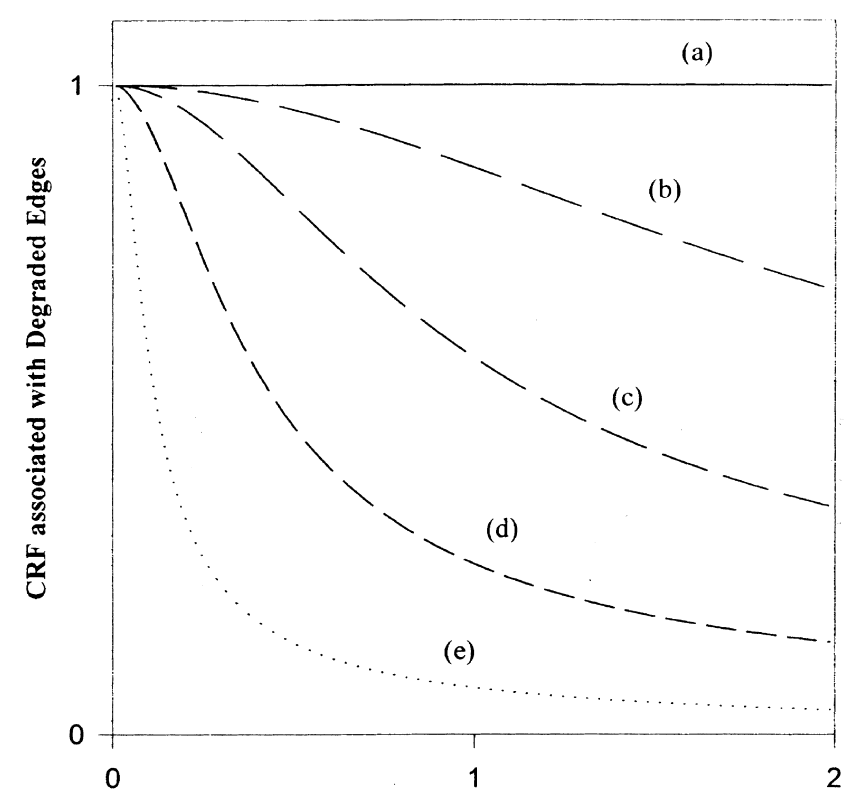

Spatial Frequency ( $u$ in $\left.\mathbf{m m}-{ }^{1}\right)$

Fig. 11. The corresponding CRF obtained by normalizing the MTF associated with an experimental edge (with a certain degradation) with respect the theoretical perfect system MTF $(\tau=0)$. geometrical shadow. For an incoherently illuminated system, the oscillations are not remarkable and only small fluctuations appear mainly due to the numerical behavior of the Bessel functions. The transition between zero intensity (black) and the maximum intensity (white) is smooth, depending on the degradation parameter. The value of the intensity at the origin of the edge-image is higher than the edge-object. There is a zone for negative values of the argument where the edgeimage intensity is not zero whereas in the edge-object intensity it is null. Then, a transition region appears in the edgeimage distribution while in the edge-object distribution there is an abrupt change. The area defined under this zone is the geometrical shadow region of the edge-image. The geometrical shadow region appears to be small for high degraded edges. However, these edges show such a large variety of gray levels that they do not have a high contrast and, therefore, they seem to be embedded on the geometrical shadow. As a consequence, they cannot be reconstructed since these high degraded edges are images with low quality and low definition. This is an important feature in the design of an image quality assessment procedure in terms of the contrast degradation.

The other magnitude referred to the quality of the edge is the associated $L S F\left(L S F_{\mathrm{d}}\right)$. The spreading shape and the central peak value are two features that characterize the edge quality. The more spreading the $L S F_{\mathrm{d}}$, the less concentrated the energy, and the smaller the edge quality. The $L S F$ could also be useful to establish resolution criteria [33] in the case of defocusing.

The shape of the associated $\operatorname{MTF}\left(M T F_{\mathrm{d}}\right)$ provides a mechanism to analyze the edge processing of the system

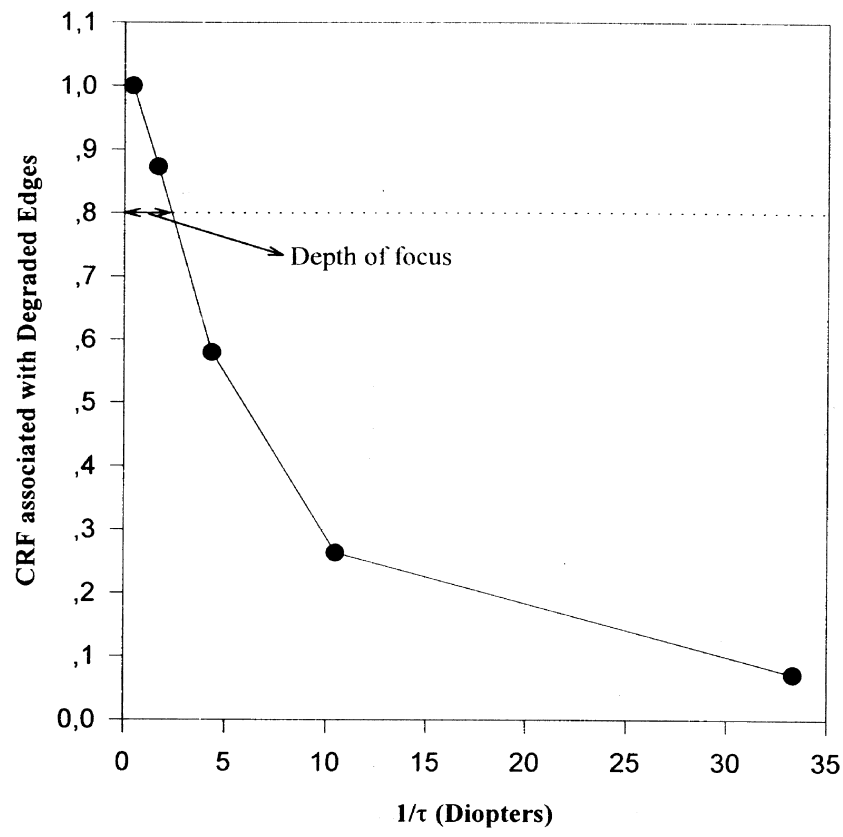

Fig. 12. $C R F$ in terms of defocusing $(\tau)$. The range of defocusing for which the $C R F$ surpass the $80 \%$ of its maximum value determines the depth of focus of the system, in dioptric units $\left(\mathrm{cm}^{-1}\right)$. The distribution corresponds to a fixed normalized spatial frequency $u=1$. 
in terms of a spatial frequency carrier and to measure its contrast degradation. As Fig. 6 shows, the $M T F_{\mathrm{d}}$ shape for $\tau$ $=1 \mathrm{~mm}$ exhibits higher contrast to all the frequencies $(0<u$ $<2$ ) than for $\tau>1 \mathrm{~mm}$, although both decrease to zero for $u$ $=2$. For $\tau \gg 1 \mathrm{~mm}$, the shape of the $M T F_{\mathrm{d}}$ is relatively insensitive to dramatic increasing of the degradation parameter: all shapes remain similar. This feature is expected since it is obvious that, having a map formed by different high degraded edges, a discrimination is not easily performed. Therefore, they should be processed similarly in the processing system, since they have analogous contrast. The resemblance between the $M T F$ of a perfect edge and the $M T F_{\mathrm{d}}$ associated with a degraded edge, and the differences of their parameters (cut-off frequency, slope) are useful, for example, to study the edge quality definition of photodetector systems (as CCD cameras) and they can provide a merit function to characterize the system [34]. The introduction of the contrast reduction function gives a cue to study the contrast as a consequence of degradation. By degrading the edge-object, one realizes that the contrast diminishes until the system is not able to give any information of the processed spatial frequencies. For $\tau \geq 2$ and medium frequencies, the contrast value is under 0.1 , that is a very low contrast and represents more a quite uniform degree of gray levels than an edge.

Relation of a contrast sensitivity function $(C S F)$ with the $C R F$ needs a deeper analysis. We know that the $C S F$ is defined as the inverse of the contrast required to produce a threshold response, referred to as Michelson contrast or contrast modulation. The inverse of a CSF has been demonstrated to be a useful tool for noise quantization in image compression, in the sense of obtaining compressed images visually indistinguishable from uncompressed ones [35]. In our present model we have seen that the distinct behavior of the experimental $C R F$ as compared with the theoretical one (for which no effects of the real image processing system are implied), comes from the influence of two parameters: the noise level (edge opacity), and a diffusion mechanism (reduction of the intensity level), producing, respectively, the equivalent to a compression and a reduction of the gray levels that define the edge degradation [36]. Consequently, a $C S F$ associated with an experimental $C R F$ will carry information only in these two parameters. It is obvious that one cannot extend the present analysis to a CSF related to human visual response to edge degradation, since we cannot define the experimental conditions to run a psychophysical test, such as luminance background and image size. We have preliminary results (not displayed here), from which one can extract a degradation sensitivity curve showing a certain constancy with degradation, but this result could be due to the actual short range of degradation, constrained by the image size (monitor specifications) [37].

No discussion of characterization of degraded edges and contrast reduction will be complete without a discussion of the human $C S F$, if one of our (ultimate) aims is to model a possible mechanism of the human spatial visual system. The human MTF and the human CSF have been studied in great detail in both normal and diseased eyes since the first publications of Campbell et al. [38,39]. Spatial and temporal CSF measurements are widely used in vision research. It should be noted that the traditionally measured CSF is not directly related to the $M T F$ of the eye, owing to the low spatial frequency fall off (presumably due to neural factors), and the band pass characteristics of the CSF. The basic limitation of the CSF technique is that it represents a complex system with many known non-linearities by a simple set of points. However, the predictive abilities of using the $C S F$ suggest that under many conditions the treatment of the visual system as a quasi-linear system is a reasonable assumption. For example, Blakemore and Campbell [40] were able to show the existence of human spatial frequency selective channels, giving rise to channel models of spatial vision (e.g. Wilson et al. [41,42]). Lang et al. [43] using a phenomenological technique based on an in vitro $O T F$ and Lakshminarayanan et al. [44], with a model of the human threshold detection, were able to predict clinical data in patients implanted with multifocal intraocular lenses. Barten [45] has evaluated subjective image quality using square root integral method and an analytical expression for the CSF.

Reduction of contrast has deleterious effects on normal (and abnormal) visual performance in real world environments [46] and in activities such as reading [47,48] and driving [49]. Using the CSF, advances have been made for characterizing the perception of degraded images due to noise and blur [50,51,26] and in letter recognition [52]. $C S F$ measures have also been used for image processing applications [53-55].

Detailed discussion of the $C S F$, its measurement and implications as well as the role of contrast human vision are beyond the scope of this discussion. However, we refer the reader to the many books and articles available on this subject [53-55]. A future goal of our research would be to relate the formalism presented herein to the human CSF.

The implications of the model to give a tolerance limit in terms of the degradation parameter, that is, a resolution criterion, are the subject of present research. We are concerned also in the task of interpreting more complicated systems, characterized by degraded $L S F$ [56,57] which implies a degraded system with a degraded $M T F$. In this sense, defining a $C R F$ seems more complex, as one has to compare two degraded $M T F \mathrm{~s}$, the one associated with a degraded edge, and the $M T F$ of a degrading system. These aspects are under development.

\section{Acknowledgements}

Partial results of this work were presented at the IVth National Meeting of the Spanish Optical Society, SEDO, Granada, Spain, September 1994; the 17th Congress of the 
International Commission for Optics (ICO), Taejon, Korea, August 1996; and the Annual Meeting of the Optical Society of America, Long Beach, USA, October 1997. The financial support of the Spanish Ministry of Health, the Institute of Health 'Carlos III' and the Health Research Foundation, under project 95/1518, and project PR160/934829/93 from the Rectorate of the Complutense University are acknowledged. We are indebted to Ivan Sanz Rodriguez for his collaboration. Also, Carmen Bravo gave us computational assistance at the Computing Center of the Complutense University of Madrid. We thank Professor J.M. Enoch for helpful suggestions.

\section{References}

[1] K.S. Shanmugam, F.M. Dickey, J.A. Green, An optimal frequency domain filter for edge detection, IEEE Trans. Patt. Anal. Mach. Intell. 1 (1) (1979) 37-49.

[2] M. Basu, Gaussian derivative model for edge enhancement, Pattern Recog. 27 (11) (1994) 1451-1461.

[3] C.S. Williams, O.A. Becklund, Introduction to the optical transfer function, Wiley Series in Pure and Applied Optics, Wiley, New York, 1989, pp. 57-59.

[4] L. Yaroslavsky, The theory of optimal methods for localization of objects in pictures, in: M.L. Calvo and M. Chevalier (Eds.), Fundamentals, Applications and Analysis of Digital Image Processing, Book Notes, Spanish Optical Society (SEDO), CSIC, Madrid, 1994, pp. 70-272.

[5] F. Bergholm, Edge focusing, IEEE Trans. Pattern Anal. Mach. Intell. 9 (6) (1987) 726-740.

[6] V. Kayargadde, J.B. Martens, Perceptual characterization of images degraded by blur and noise: model, J. Opt. Soc. Am. A 13 (6) (1996) $1178-1188$.

[7] V. Kayargadde, J.B. Martens, Perceptual characterization of images degraded by blur and noise: experiments, J. Opt. Soc. Am. A 13 (6) (1996) 1166-1177.

[8] V. Kayargadde, J.B. Martens, Estimation of perceived image blur using edge features, Int. J. Imaging Systems Technol. 7 (1996) $102-109$.

[9] R.M. Shapley, D.J. Tolhurst, Edge detectors in human vision, J. Physiol. (Lond.) 229 (1973) 165-183.

[10] J.J. Kulikowski, P.E. King-Smith, Spatial arrangement of line, edge and grating detectors revealed by subthreshold summation, Vision Res. 13 (1973) 1455-1478.

[11] D. Marr, E. Hildreth, Theory of edge detection, Proc. R. Soc. Lond. B207 (1980) 187-217.

[12] J. Gaskill, Linear Systems, Fourier Transforms and Optics, Wiley, New York, 1978, chap. 11.

[13] E.W. Marchand, Derivation of the point spread function from the line spread function, J. Opt. Soc. Am. 54 (1964) 915-919.

[14] W. Weinstein, Light distribution in the image of an incoherent illuminated edge, J. Opt. Soc. Am. 44 (8) (1954) 610-615.

[15] R. Barakat, A. Houston, Line spread and edge spread functions in the presence of off-axis aberrations, J. Opt. Soc. Am. 55 (1965) 11321135.

[16] W. H. Swanter, Ch. R. Hayslett, Point spread functions, edge response and modulation transfer functions of obscured aperture optical systems, Research Projects Office, Technical Memorandum, USA, 1975.

[17] M.L. Calvo, A. Manzanares, M. Chevalier, V. Lakshminarayanan, A formalism for analyzing degraded edges using modified Heaviside functions, in: V. Lakshminarayanan (Eds.), Basic and Clinical
Applications of Vision Science, Kluwer Academic, Dordrecht, 1997, pp. 77-81.

[18] A.P. Pentland, A new sense for depth of field, IEEE Trans. Pattern Anal. Mach. Intell. 9 (4) (1987) 523-531.

[19] J.A. Marshall, Ch.A. Burbeck, D. Ariely, J.P. Rolland, K.E. Martin, Occlusion edge blur: a cue to relative visual depth, J. Opt. Soc. Am. 13 (4) (1996) 681-688.

[20] B.N. Begunov, N.P. Zakaznov, S.I. Kiryushin, V.I. Kuzichev, Optical Instrumentation Theory and Design, Mir, Moscow, 1988, chap. 6, Section 6-2.

[21] H. Struve, Wied. Ann. 47 (1882) 1008.

[22] W.H. Steel, Calcul de la répartition de la lumière dans l'image d'une ligne, Revu. Opt. 31 (7) (1952) 334-340.

[23] A. Manzanares, M.L. Calvo, M. Chevalier, V. Lakshminarayanan, W.H. Line spread Function proposed by, Steel: a revision (Technical Note), Appl. Opt. 36 (19) (1997) 4362-4366.

[24] M. R. Spiegel, L. Abellanas, Fórmulas y tablas de matemática aplicada, McGraw-Hill, New York, 1988.

[25] Gradshteyn, I.M.R., in: A. Jeffrey (Ed.), Table of Integrals, Series and Products, Academic Press, New York, 1994.

[26] G.E. Legge, K.T. Mullen, G.C. Woo, F.W. Campbell, Tolerance to visual defocus, J. Opt. Soc. Am. A 4 (1987) 851-863.

[27] E.W. Marchand, From line to point spread function: the general case, J. Opt. Soc. Am 55 (4) (1964) 352-355.

[28] R. Barakat, A. Houston, Line spread function and cumulative line spread function for systems with rotational symmetry, J. Opt. Soc. Am. 54 (1964) 768-773.

[29] M. Abramowitz, I. Stegun, Handbook of Mathematical Functions, Dover, New York, 1968, p. 556.

[30] J. Rabin, Luminance effects on visual acuity and small letter contrast sensitivity, Optom. Vision Sci. 71 (11) (1994) 685-688.

[31] P. Artal, R. Navarro, Monochromatic modulation transfer function of the human eye for different pupil diameters: an analytical expression, J. Opt. Soc. Am. A 11 (1) (1994) 246-249.

[32] S. Marcos, E. Moreno, R. Navarro, Depth of field of the human eye, in: Proceedings of the Vth Optical National Meeting, Valencia, Spain, 1997, pp. 335-336 (in Spanish).

[33] M.L. Calvo, M. Chevalier, V. Lakshminarayanan, P.K. Mondal, Resolution criteria and modulation transfer function (MTF)/line spread function (LSF) relationship in diffraction limited systems, J. Opt. 25 (1) (1996) 1-21.

[34] A. Simon, E. Shaw, M.L. Calvo, Merit functions of CCD cameras based on edge image processing, in: J.S. Chang, J.H. Lee, S.Y. Lee anad C.H. Nam (Eds.), 17th Congress of the International Commission for Optics: Optics for Science and New Technology, Proceedings of SPIE, vol. 2778, 1996, 65-66.

[35] S. Daly, Application of a noise-adaptive contrast sensitivity function to image data compression, Opt. Engng 29 (8) (1990) 977-987.

[36] I.Sanz, M.L. Calvo and M. Chevalier, Digitally implemented soft edges with predetermined luminance: an analysis of its influence on image degradation, in: E. Wenger et al. (Eds.), Digital Image Processing and Computer Graphics (DIP-97) Proceedings of SPIE, vol. 3346, 1998, 72-83.

[37] M.L. Calvo, I. Sanz, M. Chevalier, V. Lakshminarayanan, A psychophysical test based on degraded edge imaging: contrast sensitivity and threshold luminance, in: A. Sanfeliu, J.J. Villanueva, J. Vitriá (Eds.), Proceedings of the VIIth National Symposium on Pattern Recognition and Image Analysis UAB, Barcelona, vol. 2, 1997, pp. 118-119.

[38] F.W. Campbell, R.W. Gubisch, Optical quality of the human eye, J. Physiol. Lond. 186 (1966) 558-578.

[39] F.W. Campbell, J.G. Robson, Applications of Fourier analysis to the visibility of gratings, J. Physiol. Lond. 197 (1968) 551-566.

[40] C. Blakemore, F.W. Campbell, On the existance of neurons in the human visual system selectively sensitive to the orientation and size of retinal images, J. Physiol. Lond. 203 (1969) 237-260.

[41] H.R. Wilson, J.R. Bergen, A four-mechanism model for threshold spatial vision, Vision Res. 19 (1979) 19-32. 
[42] H.R. Wilson, D.J. Gelb, Modified line element theory for spatial frequency and width discrimination, J. Opt. Soc. Am. A 1 (1984) $124-131$.

[43] A. Lang, V. Lakshminarayanan, V. Portney, A phenomenological model for interpreting the clinical significance of the in-vitro OTF, J. Opt. Soc. Am. A 10 (1995) 1600-1610.

[44] V. Lakshminarayanan, A. Lang, V. Portney, The 'expected visual outcome' model: methodology and clinical validation, Optom. Vision Sci. 72 (1995) 511-521.

[45] P.G.J. Barten, Evaluation of subjective image quality with the squareroot integral method, J. Opt. Soc. Am. A 7 (1990) 2024-2031.

[46] C. Owsley, M. Sloane, Contrast sensitivity, acuity and the perception of real world targets, Br. J. Ophthal. 71 (1987) 791-796.

[47] G.E. Legge, Three perspectives on low vision reading, Optom. Vision Sci. 68 (1991) 763-769.

[48] G.E. Legge, G.S. Rubin, A. Luebker, Psychophysics of reading-V. The role of contrast in normal vision, Vision Res. 27 (1987) 1165-1177.

[49] W. Adrian, Visibility of targets: model for calculation, Lighting Res. Technol. 21 (1989) 181-188.

[50] V. Lakshminarayanan, A. Lang, The relationship between defocused MTF and spatial frequencies needed for letter recognition, in: Vision Science and its Applications. Technical Digest, vol. 1, Optical Society of America, Washington DC, 1995, pp. 159-162.
[51] K.N. Ngan, H.C. Koh, W.C. Wong, Hybrid image coding scheme incorporating human visual system characteristics, Opt. Engng 30 (7) (1991) 940-947.

[52] L.A. Saghri, P.S. Cheatham, A. Habibi, Image quality measure based on a human visual system model, Opt. Engng 28 (7) (1989) 813-819.

[53] R.L. DeValois, K.K. DeValois, Spatial Vision, Oxford University Press, New York, 1988.

[54] R.Shapley, D.Man-Kit Lam, Contrast Sensitivity, MIT Press, Cambridge, MA, 1993.

[55] M.P.Nadler, D.Miller, D.J.Nadler, Glare and Contrast Sensitivity for Clinicians, Springer, Heidelberg, 1990.

[56] M.L. Calvo, M. Chevalier, V. Lakshminarayanan, A. Manzanares, Analysis of the Heaviside function as a systematic generator of lines and edges: failure on LSF/MTF reciprocity and sampling, in: Proceedings of the IVth National meeting of the Spanish Optical Society (SEDO), Granada, Spain, 1994, pp. 29-30 (in Spanish).

[57] M.L. Calvo, A. Manzanares, M. Chevalier, V. Lakshminarayanan, Edge image processing: an analysis of modified Heaviside functions as degraded edge generators, in: J.S. Chang, J.H. Lee, S.Y. Lee, C.H. Nam (Eds.), Proceedings of the 17th Congress of the International Commision for Optics: Optics for Science and New Technology, Proceedings of SPIE, vol. 2778, 1996, pp. 63-64. 